\title{
The Association of Clinical Pathologists: 89th general meeting
}

The 89th general meeting of the Association of Clinical Pathologists was held at Imperial College, London, on 28 and 29 September 1972 under the Presidency of Sir James Howie, whose Presidential Address is printed on page 921. On this occasion there were five symposia: 'The lymphoreticular system', 'Decompression sickness', 'Chromosomes and disease', 'Encephalitis and meningitis', and 'Equipment for haematology'. Abstracts of papers read at these meetings and at the other sessions follow.

\section{Scientific communications I}

\section{A Histological Analysis of Granulomatous Hepatitis} M. HUGHeS AND H. FOX (Department of Pathology, University of Manchester) A study has been made of 33 cases of granulomatous hepatitis. Of these, 12 were eventually established as cases of sarcoidosis, nine as cases of tuberculosis, and four as examples of primary biliary cirrhosis. No clinical diagnosis was ever reached in six cases.

Analysis of the histological features of these cases shows that the presence of caseation or of tubercle bacilli will only allow for the diagnosis of approximately $50 \%$ of cases of tuberculosis. Histological features which are suggestive of tuberculosis are a paucity of granulomata, a scantiness of giant cells, a relatively mild surrounding chronic inflammatory cell infiltrate and an absence of reticulin. The granulomata are more likely to be due to sarcoidosis if they are numerous, contain many giant cells, are confined to the portal areas, and show a well marked surrounding non-specific chronic inflammatory cell infiltrate.

Histological features of cases in which no clinical diagnosis was reached suggest that they are more likely to be due to sarcoidosis than to tuberculosis.

\footnotetext{
A Case of Brucella Arthritis in the Wrist J. L. BRENNAN AND C. A. MORRIS (Robert Jones and Agnes Hunt Orthopaedic Hospital, Oswestry and Public Health Laboratory, Shrewsbury) A farmer aged 48 presented in August 1971 with a history of pain in the right wrist for six weeks. There was swelling of the dorsum and radial side of the wrist. A provisional diagnosis of tenosynovitis was made and the wrist was immobilized in plaster for six weeks. In October the patient was discharged as he was by then free of pain and swelling, but he returned in February 1972 with a recurrence.
}

Radiographs of the wrist, which previously had shown no obvious bony abnormality, now revealed a destructive lesion of the hamate bone. Tuberculosis, rheumatoid arthritis, and brucellosis were considered in the differential diagnosis. The Brucella abortus agglutination test was positive in phenol saline and in mercaptoethanol at $1 / 400$ and the Brucella CFT positive at $1 / 320$.

The wrist was explored surgically. A synovial biopsy showed subacute inflammation with appearances intermediate between an acute pyogenie infection and classical tuberculosis. Typical epf thelioid systems and caseation were absent, but is defined epithelioid cells and one Langhans type giant cell were seen. The bone showed similar tissue in the marrow spaces with osteoclastic activity. Brucella abortus biotype 1 was cultured from the synovium. A diagnosis of brucella osteomyelitis and synovitis was made. The patient gave a history of brucellosis among his cattle.

Helminthic Pseudotumours of the Bowel P. P. ANTHONY (Middlesex Hospital Medical School, London) Human infestation with nematode worms of the superfamily Strongyloidea may give rise to serious surgical complications. In this communication 34 cases are presented from Uganda where the condition appears to be common but it is also known to occur in other parts of Africa, the Far East, and the Americas. Worms of the genus Oesophagostomum are most frequently responsible. These are common parasites of ruminants, apes, and monkeys in which their histotropic phase is confined to the $\tilde{O}$ bowel wall. Development in man is abnormal and N results in persistence and further migration of the parasite outside the bowel wall. Large tumour-like masses and abscesses form, most commonly in the $\stackrel{\mathbb{D}}{\overparen{D}}$ ileocoecal angle, where they are usually misdiagnosed $\stackrel{\oplus}{?}$ as carcinoma, tuberculosis, or appendicular abscess, 0 particularly in expatriate Europeans. All ages and 